\title{
Mann ist Mann
}

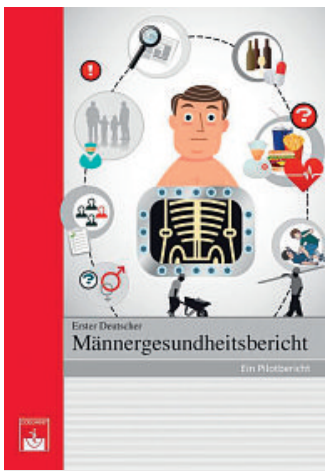

Der Männergesundheitsbericht zeigt es: Der Vorsprung der Frauen bei der Lebenserwartung nimmt ab.
1 Bardehle D, Stiehler M (Hrsg.). Erster Deutscher Männergesundheitsbericht. München: Zuckschwerdt. 2010;
Das waren noch Zeiten, als Männer Drachen bezwangen oder auf der Titanic die Rettungsboote den Frauen und Kindern überliessen. Das Bild der Helden und Ritter hat sich in der Dienstleistungsgesellschaft gründlich gewandelt. Das Mannsein ist in jedem Alter manifest schwieriger geworden. Das bisher starke Geschlecht leidet an Orientierungskrisen, schluckt Ritalin, mutiert zum jugendlichen Schläger, wählt später rechtspolitische Parteien, kämpft um das elterliche Sorgerecht und flüchtet in ein Väterhaus, wenn Femokratinnen und Linke wieder einmal zuschlagen. Dieser Eindruck entsteht, wenn man den täglichen Medienberichten glauben will.

Schlechte Spermien, Weichmacher und Pestizide im Urin oder Wodkaleichen in der ehemaligen Sowjetunion; um die virile Gesundheit steht es schlecht. Die unterschiedliche Lebenserwartung der Geschlechter ist seit Jahrzehnten ein Forschungsthema. «Eine Krankheit namens Mann» titelte vor Jahren der Spiegel, der Mann, ein «beratungsresistenter Gesundheitsidiot» und ein «gesundheitlicher Sanierungsfall», unterstreichen andere Autoren. Lange erwartet, spricht «Der erste Deutsche Gesundheitsbericht» aus dem Jahr 2010 endlich Klartext [1]. Experten unterschiedlicher Lebensbereiche erörtern darin die Defizite der männerspezifischen Gesundheit und deren Förderung. Den 198-seitigen Pilotbericht ermöglicht haben die «Stiftung Männergesundheit» und die «Deutsche Gesellschaft für Mann und Gesundheit», unterstützt von der «Deutschen Krankenversicherung DKV». Zur Debatte stehen die unterschiedliche Arbeitsteilung und das traditionelle Leitbild von Männlichkeit. Soldatische Tugenden als Orientierungsmuster erfolgreicher Männer seien nicht mehr gefragt, und das historisch geprägte Schädigungsverhalten erfordere noch viel Erziehung. Frauen leben in Deutschland 5,3 Jahre länger; eine höhere Lebenserwartung erreichen sie nur noch in Norwegen, den Niederlanden, in Italien, Frankreich, Schweden, der Schweiz, Spanien und dem Vereinigten Königreich.

Als erste Massnahme empfehlen Fachleute die Senkung der vorzeitigen Sterblichkeit, verursacht durch Lungenkrebs, ischämische Herzkrankheiten, Hypertonie, Krankheiten der Leber, Depressionen, Gewalt, Unfälle und Suizide. Für jede Altersgruppe werden Risiken und Probleme statistisch erfasst und kommentiert. Die Klosterforschung hat mittlerweile belegt, dass bei sehr ähnlichen Lebensbedingungen, Mönche und Nonnen eine fast gleiche Lebenserwar- tung haben. Der genetische Vorteil der Frauen macht aber nur ein Jahr an erhöhter Lebenserwartung aus, der grössere Unterschied wird auf kulturelle und soziale Ursachen zurückgeführt. Zum Beispiel auf Bedingungen am Arbeitsplatz, die ökonomisch forcierte Individualisierung, die Zwänge der Globalisierung, Arbeitslosigkeit, Migrationshintergründe und die Geschlechterblindheit der Medizin. «Nicht nur durch die Entwicklung der PDE-5-Inhibitoren, sondern mehr noch durch die Gesundheitsstatistiken fiel auf, dass auch Männer spezifische Probleme haben, die Beachtung verdienen.» Männer seien mit ihren Problemen zu wenig im Blick, es fehle an validen Daten, an Forschung und an präventiven und kurativen Angeboten. «Eine spezifisch auf Männer bezogene Gesundheitsforschung und Praxis ist im Vergleich zu den Aktivitäten auf Frauenseite in der Schweiz nach wie vor die Ausnahme», stellte die schweizerische Gesundheitsstiftung RADIX schon 2005 fest. Es sei aber wichtig die gesundheitlichen Interessen von Männern und Frauen nicht gegeneinander auszuspielen. Die Vielfalt gelebter Männlichkeit und Weiblichkeit müsse im Alltag die gleichen Chancen haben.

Das Bundesamt für Statistik hat die Sterblichkeit der Geburtsjahrgänge 1900 bis 2008 analysiert und daraus ein mathematisches Modell bis zum Jahr 2030 entwickelt. Alle drei Jahre hat die durchschnittliche Lebensdauer um ein Jahr zugenommen. Die Frauen sind Rekordhalterinnen, doch ihr Vorsprung zeigt im Laufe der Generationen eine abnehmende Tendenz. Die Männer holen ihren Rückstand allmählich auf, so dass ihr Gewinn an Lebensjahren seit über vierzig Geburtsjahrgängen jenen der Frauen übersteigt. «Vive la difference», vielleicht ist sie auch hier nicht so gross, wie es ein lukrativer, kosmetischer und pharmazeutisch-medizinischer Markt suggeriert. Mann ist Mann, besonders hierzulande, wo das Gewehr im Besenschrank die wacklige Identität des wehrhaften Familienvaters kräftigt. Allen männerbündlerischen Protestbewegungen und antifeministischen Demonstrationen zum Trotz: Das Patriarchat sitzt nach wie vor fest im Sattel. Der gesellschaftliche Wandel ist eine Tatsache, er verläuft aber viel komplexer, als es Gesundheitsberichte von Männern und Frauen zu erfassen vermögen. Dennoch ist ihre Wirkung nicht zu unterschätzen, besonders wenn Ärzte und Ärztinnen sie ernst nehmen.

Erhard Taverna 